\title{
Case Report \\ Effects of Adenotonsillectomy on Neurocognitive Function in Pediatric Obstructive Sleep Apnea Syndrome
}

\author{
Fumie Horiuchi, ${ }^{1}$ Yasunori Oka, ${ }^{2,3}$ Kenjiro Komori, ${ }^{1,4}$ Yasumasa Tokui, ${ }^{1,3}$ \\ Teruhisa Matsumoto, ${ }^{1}$ Kentaro Kawabe, ${ }^{1}$ and Shu-ichi Ueno ${ }^{1}$ \\ ${ }^{1}$ Department of Neuropsychiatry and Neuroscience, Ehime University Graduate School of Medicine, \\ Shitsukawa, Toon-city, Ehime 791-0295, Japan \\ ${ }^{2}$ Center for Sleep Medicine, Ehime University Hospital, Ehime, Japan \\ ${ }^{3}$ Hiroshima Sleep Center, Hiroshima, Japan \\ ${ }^{4}$ Zaidan Niihama Hospital, Niihama, Ehime, Japan \\ Correspondence should be addressed to Fumie Horiuchi; matsufu@m.ehime-u.ac.jp
}

Received 24 February 2014; Revised 28 April 2014; Accepted 12 May 2014; Published 26 May 2014

Academic Editor: Samuele Cortese

Copyright ( $\odot 2014$ Fumie Horiuchi et al. This is an open access article distributed under the Creative Commons Attribution License, which permits unrestricted use, distribution, and reproduction in any medium, provided the original work is properly cited.

Obstructive sleep apnea syndrome (OSAS) in children does not only present with symptoms of sleep disturbances but also with associated symptoms such as growth failure, enuresis, academic learning difficulties, and behavioral problems, including attention deficit/hyperactivity disorder- (ADHD-) like symptoms. We evaluated neurocognitive functions before and after adenotonsillectomy in a patient with OSAS. An 11-year-old boy suspected of having ADHD with nocturnal enuresis was referred for evaluation. He was found to have adenotonsillar hypertrophy. Presence of snoring was evident only after detailed medical interview. Polysomnography confirmed the diagnosis of OSAS, which was subsequently treated by adenotonsillectomy. The apnea/hypopnea index decreased from 21.9 at baseline to 1.8 after surgery, and the frequency of enuresis fell from almost nightly to 2-3 times per month. Neurocognitive and behavioral assessment after the treatment of OSAS showed significant improvement in cognitive functions, especially attention capacity and considerable amelioration of behavioral problems including ADHD-like symptoms. As the most common cause of pediatric OSAS is adenotonsillar hypertrophy, medical interview and oropharyngeal examination should always be performed in children suspected of having ADHD. The necessity of sleep evaluation for children with ADHD-like symptoms was also emphasized.

\section{Introduction}

Obstructive sleep apnea syndrome (OSAS) is a common disorder in adults as well as in children. The prevalence of pediatric OSAS is up to $5.7 \%$ [1-3]. The clinical symptoms of pediatric OSAS are not always compatible with those of adult OSAS. A wide range of symptoms and signs are associated with pediatric OSAS in the developmental stages. Diagnosis of OSA in children is made on the basis of sleep history, physical examination, and polysomnographic findings. In the diagnostic criteria of pediatric OSAS by the International Classification Sleep Disorder, second edition (ICSDII), witnessed snoring and/or labored/obstructed breathing during the child's sleep is required. In addition, the caregiver reporting at least one of the following is necessary: paradoxical inward rib-cage motion during inspiration, movement arousals, diaphoresis, neck hypertension during sleep, excessive daytime sleepiness/hyperactivity, aggressive behavior, morning headaches, and secondary enuresis [4]. Untreated pediatric OSAS has been related with various problems such as attention deficit/hyperactivity disorder (ADHD), poor academic achievement, and behavioral problems [5]. The most common cause of pediatric OSAS is adenotonsillar hypertrophy and the peak age of pediatric OSAS is 3-7 years, which parallels the age of adenotonsillar enlargement [6].

ADHD is a neuropsychiatric syndrome affecting 3-16\% of school children with world prevalence of 5.29\% [7]. The diagnosis of ADHD is sometimes difficult because other 
psychiatric and medical disorders such as anxiety disorder and sleep disorder can also mimic ADHD symptomatology [8].

There is a well-established correlation between sleep disturbances and ADHD [9]. In the case of pediatric OSAS due to adenotonsillar hypertrophy, the surgery may make it possible to improve behavioral and cognitive performance, although parents and even physicians do not necessarily associate these behavioral and neurocognitive deficits with OSAS.

The aim of this study was to investigate the necessity of identifying pediatric OSAS for children with ADHD symptomatology and to assess the effects of treatment of OSAS on neurocognitive functions.

\section{Case Presentation}

An 11-year-old boy was referred to the university hospital with suspected ADHD. He tended to be hyperactive since he started crawling, and he has been still fidgeting at school, with academic problems related to inability to concentrate on work. The socioeconomical level of his family was on average. ADHD was suspected by teachers based on forgetfulness of school needs and homework. His symptoms were compatible with the diagnostic criteria of ADHD, predominantly inattentive type, based on the diagnostic criteria of Diagnostic and Statistical Manual, fourth edition, text revision (DSMIV-TR) [10]. The medical history also included nocturnal enuresis almost every night, treated with oxybutynin and desmopressin, though both medications were ineffective.

Physical examination showed height within the normal range $(155 \mathrm{~cm})$, body weight of $48.4 \mathrm{~kg}$, and normal body mass index $\left(20.0 \mathrm{~kg} / \mathrm{m}^{2}\right.$, Rohrer index: 129.2). His parents had not noticed his snoring until they had the detailed medical interview. Oropharyngeal examination showed adenotonsillar hypertrophy with Mackenzie stage II to stage III. Presence of snoring was evident only after medical interview to the parents. Based on suspicion of OSAS, the patient was scheduled for attended polysomnography (PSG) with video monitoring and multiple sleep latency test (MSLT). PSG recording included electroencephalography (EEG) (C3A2 and C4-A1), electrooculography, submental and bilateral lower limb electromyography, and electrocardiography, together with continuous recording of oronasal airflow, chest and abdominal movements, and oxygen saturation. Sleep stages were scored according to the Rechtschaffen and Kales manual [11]. Apneas were scored when the drop of flow by $\geq 90 \%$ of preevent baseline for at least the duration of 2 breaths. Hypopneas were scored when the signal dropped by $\geq 30 \%$ for at least the duration of 2 breaths with $\geq 3 \%$ oxygen desaturation. All-night PSG showed frequent episodes of obstructive sleep apnea/hypopnea (apnea/hypopnea index = 21.9/hour) associated with falls in oxygen saturation and EEG arousals.

He also underwent a series of neurocognitive tests and his parents filled out questionnaires. The cognitive test battery consisted of the Japanese version of the Wechsler Intelligence Scale for Children-Third Edition (WISC-III), Kaufman
TABLE 1: Polysomnographic data before and after treatment.

\begin{tabular}{lcc}
\hline & Pretreatment & Posttreatment \\
\hline Polysomnography & & \\
Total recording time (min) & 537 & 497 \\
Total sleep time (min) & 494 & 477 \\
Sleep time (min) & 512 & 490 \\
Sleep efficiency (\%) & 89.6 & 96.4 \\
Sleep latency (min) & 27 & 4 \\
REM latency (min) & 91 & 110 \\
Arousal (/h) & 3.6 & 2.8 \\
Stage I NREM sleep (\%) & 8.6 & 6.9 \\
Stage II NREM sleep (\%) & 42.9 & 39.2 \\
Stage III/IV NREM sleep (\%) & 23.7 & 24.0 \\
REM sleep (\%) & 21.2 & 27.5 \\
Apnea/hypopnea index & 21.9 & 1.8 \\
Multiple sleep latency test & & \\
Sleep latency time (min) & & \\
1st nap & 20.0 & 13.5 \\
2nd nap & 18.0 & 20.0 \\
3rd nap & 5.0 & 12.5 \\
4th nap & 20.0 & 20.0 \\
5th nap & 8.0 & 20.0 \\
Mean sleep latency & 14.2 & 17.2 \\
Sleep-onset REM period & - & \\
\hline
\end{tabular}

Assessment Battery for Children (K-ABC), for assessment of intelligence and achievement, the Rey-Osterrieth Complex Figure Test (ROCF) as a scale of visuospatial construction, and the Rey's Auditory Verbal Learning Test (RAVLT) to assess verbal learning and memory [12-15]. For behavioral assessment, the parents' version of the Child Behavior Checklist (CBCL) was used to measure social competency and problematic behavior [16].

Based on the diagnosis of pediatric OSAS, he underwent adenotonsillectomy.

He was reevaluated for PSG, MSLT, and neurocognitive and neurobehavioral assessment 4 months after surgery. After adenotonsillectomy, the frequency of enuresis reduced to 2$3 /$ month and his snoring disappeared. Although he did not complain about sleepiness before the operation, he reported improvement in daytime sleepiness and concentration after adenotonsillectomy.

Table 1 lists the results of PSG and MSLT conducted before and after adenotonsillectomy. The apnea/hypopnea index decreased to 1.8 after treatment, and the mean sleep latency of MSLT increased from 14.2 minutes to 17.2 minutes. On the WISC-III, the score of FD increased significantly from 106 to 129, but no differences were noted in the scores of VIQ, PIQ, and FIQ. With regard to the 13 subtests, the scores of Arithmetic, Picture Arrangement, and Digit Span subtests showed significant improvement (Figure 1). Furthermore, the score of Simultaneous Processing Scale of the K-ABC increased from 95 (below the average score of 100) to 112 (Table 2). The immediate and delayed recall trial scores of the ROCF improved, and the recall trial scores of RAVLT also 


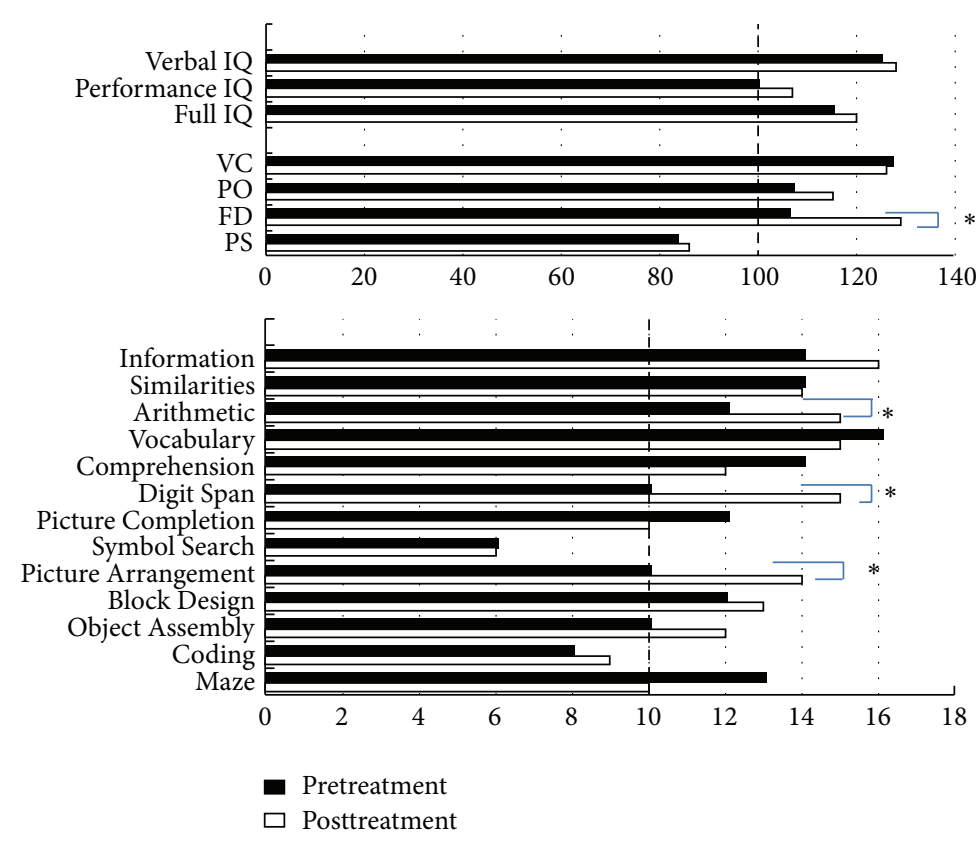

FIGURE 1: Wechsler Intelligence Scale for Children-Third Edition (WISC-III) scores of IQ, 4 factors and 13 subscores before and after treatment. Verbal IQ is calculated by the scores of "Information," "Similarities," "Arithmetic," "Vocabulary," and "Comprehension." Performance IQ is calculated by the scores of "Picture," "Comprehension," "Coding," "Picture," "Arrangement," "Block Design," and "Object Assembly." Full IQ = Verbal IQ + Performance IQ. VC (verbal comprehension) is calculated by the scores of "Information," "Similarities," "Vocabulary," and "Comprehension." PO (perceptual organization) is calculated by the scores of "Picture Comprehension," "Picture Arrangement," "Block Design," and "Object Assembly." FD (freedom of distraction) is calculated by the scores of "Arithmetic" and "Digit Span." PS (performance speed) is calculated by the scores of "Coding" and "Symbol Search". * $P<0.05$.

TABLE 2: Kaufman Assessment Battery for children (K-ABC) before and after treatment.

\begin{tabular}{lcc}
\hline & Pretreatment & Posttreatment \\
\hline Sequential Processing Scale & 123 & 125 \\
Simultaneous Processing Scale & 95 & 112 \\
Mental Processing Composite Scale & 109 & 122 \\
Achievement Scale & 125 & 124 \\
Nonverbal Scale & 93 & 100 \\
\hline
\end{tabular}

showed large increases (Table 3 ). In addition, all three general scales of the CBCL, Internalizing $\mathrm{T}$ score (from withdrawn behavior, somatic complains, and anxious/depressed), and Externalizing $\mathrm{T}$ score (from delinquency and aggression) improved from clinical range to almost normal range. Out of 8 subscale scores of the CBCL, social problems, attention, delinquency, and aggression were above clinical cutoff value (70 points) before treatment and all subscores became below the clinical cutoff values after adenotonsillectomy (Figure 2).

\section{Discussion}

The present study demonstrated that adenotonsillectomy for severe pediatric OSAS resulted in significant improvement in nocturnal ventilation, sleep architecture, snoring, neurocognitive functions, especially attention capacity, and behavioral problems, including ADHD-like symptoms.
TABLE 3: Rey-Osterrieth Complex Figure Test (ROCF) and Rey's Auditory Verbal Learning Test (RAVLT) before and after treatment.

\begin{tabular}{lcc}
\hline & Pretreatment & Posttreatment \\
\hline ROCF (/36 score) & & \\
Copy & 33.0 & 35.0 \\
Immediate recall & 11.5 & 20.5 \\
Delayed recall & 11.5 & 20.5 \\
RAVLT & $6-6-11-12-13-(4)-11$ & $7-12-15-15-15-(7)-15$ \\
Recognition & $15 / 15$ & $15 / 15$ \\
Errors & 0 & 0 \\
\hline
\end{tabular}

ROCF involves copying complex geometric figures and then reproducing them from memory, immediately and after a brief delay. It is a count of the number of parts reproduced irrespective of organizational integrity.

RAVLT involves listening to 15 words and recalling them from memory. This performance is repeated 7 times, and on the 6th trial another set of 15 words is used and the total number of correctly recalled words is scored.

ADHD in children is associated with various sleep disorders including pediatric OSAS, periodic limb movement (PLMD), enuresis, and delayed sleep phase syndrome (DSPS) [17-21]. According to a meta-analysis of both subjective and objective studies, the incidence of sleep disturbances was significantly higher among children with ADHD as compared to a control group [22]. Naseem et al. [23] suggested that sleep disorders, especially sleep apnea, might be one of the underlying causes of ADHD. 


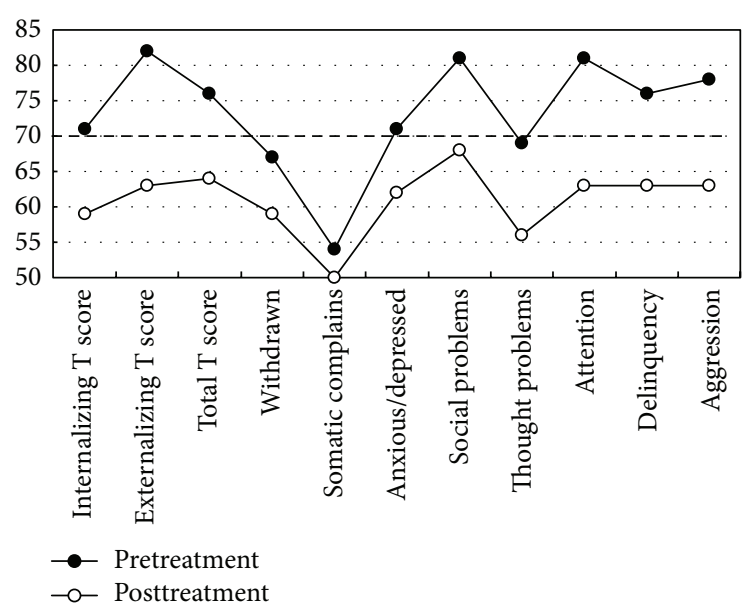

Figure 2: Child Behavior Checklist scores of general scores and subscores before and after treatment.

On the other hand, patients with sleep disturbances often display behavioral patterns that resemble some features of ADHD. Parents of children with OSAS tend to focus solely on daytime behavior problems, inattentiveness, and academic failure, rather than sleep problems. Therefore, these children are prone to be diagnosed with neurodevelopmental disorders, such as ADHD. Our patient was referred to the hospital with suspected ADHD, not OSAS, and initially fulfilled the diagnostic criteria of ADHD. It is often difficult to reveal which of the disorders is the primary and which is a byproduct of the other [9]. Moreover, in this case, parents were not aware of snoring before being pointed out by the physician. According to Chervin et al, snoring was strongly predictive of a future diagnosis of hyperactivity over the long-term [24], while children with primary snoring had a higher risk for hyperactive and inattentive behavior, compared to children who never snored [25]. Sleep is not always assessed in children suspected of having ADHD, but children with ADHD should be assessed for quality of sleep, checked for enlarged tonsils, and interviewed for presence of snoring. Sleep studies should be taken into consideration in children suspected of having OSAS as an underlying cause of ADHD-like symptoms. The use of stimulants is the most common therapeutic trial for ADHD. However, treatment of the underlying sleep disorder can avoid unnecessary use of potent and sometimes harmful medications [26].

The most common cause of nocturnal enuresis is neurological-developmental delay, but pediatric OSAS is occasionally associated with enuresis [27]. Brouilette et al. [28] reported a higher incidence of enuresis in children with OSAS compared with the control group. They also reported that $35 \%$ of children with adenohypertrophy suffered from enuresis and that adenotonsillectomy significantly improved enuresis. The high prevalence of enuresis in children with OSAS is thought to be due to the effects of obstructive sleep apnea on arousal response, bladder pressure, and urinary hormone secretion [29]. In addition, enuresis is also common in children with ADHD, although the mechanism of enuresis in ADHD remains elusive. Previous studies have demonstrated a significantly increased prevalence of ADHD in children with enuresis [30]. Fifteen percent of all enuretic children were diagnosed with the full syndrome of ADHD, and $22.5 \%$ met the criteria of the ADHD inattentive subtype [31]. Children with ADHD and drug resistant enuresis should be assessed for sleep in consideration of the possibility of OSAS.

The performance on the K-ABC test can reflect the ability of a child for academic achievements [32]. In our case, adenotonsillectomy resulted in marked improvement in the Simultaneous Processing Scale. This change enabled the child to reach the original abilities and fulfilled the cognitive potential. The effects of adenotonsillectomy emphasize OSAS as the cause of underachievement [33]. Adenotonsillectomy also significantly improved the Arithmetic, Picture Arrangement, and Digit Span subtests of the WISC-III. These cognitive functions are related to attention capacity and were probably related to the academic problems before the treatment. Based on previous reports [34-37], the most frequently reported neurocognitive impairment in patients with OSAS is verbal short-term memory, attention capacity, and problemsolving strategies. In our case, FIQ, VIQ, and PIQ were not significantly different before and after adenotonsillectomy, suggesting that OSAS did not affect IQ itself. However, the ROCF and RAVLT scores improved after treatment, and such improvement could be related indirectly to the improvement in short-term memory and attention capacity. Moreover, the ROCF reflects not only visuoperceptual and visuoconstructional ability, but also executive function, especially planning and organization [38]. The executive function could also improve after treatment. Friedman et al. [33] reported improvement in the mean scores of various cognitive tests in patients with OSAS after adenotonsillectomy, to levels similar to those of the healthy controls. They concluded that the neurocognitive deficit was mostly reversible. However, at which point the impaired neurocognitive function becomes irreversible is not clear at this stage. Undoubtedly, disturbances of neurocognitive function have a significant impact on the children's physical and mental development. Further research should address the reversibility of various neurocognitive functions in OSAS.

With regard to the behavior problems, externalizing behavior problems were obviously higher than internalizing behavior problems before adenotonsillectomy. Adenotonsillectomy in this patient did not result in significant changes in externalizing and internalizing behavior problems and all subscores of CBCL became below the cutoff point of clinical level, 70. In this regard, Lewin et al. [39] reported that externalizing behavior problems in CBCL were common in patients with mild OSAS. Our results are compatible with the previous findings. Longitudinal studies of large sample size, starting at young age, are needed in order to explore the long-term consequences of OSAS in children and determine the specific effects of pediatric OSAS on neurocognitive functions.

The present study has several limitations. As we reevaluated neurocognitive functions 4 months after the treatment, practice effect with regard to the neurocognitive function tests could have influenced the results. The interval between 
the tests before and after the treatment may have been short to confirm the effectiveness of adenotonsillectomy. A randomized trial of adenotonsillectomy for childhood sleep apnea reported that after a 7-month intervention period, school-age children with prolonged oxyhemoglobin desaturation, who underwent surgery, did not show the significant improvement in attention or executive function measured by neuropsychological testing, although the improvements of behavior, quality of life, and polysomnographic findings were observed [40]. In the meta-analysis, the duration between assessment before and after adenotonsillectomy was wide range from 2.4 months to 18 months [8]. The optimal interval for assessment before and after surgery was necessary to be determined. According to Chervin et al, there could be persistent OSA at 1 year follow-up in up to $20 \%$ of patients with OSAS [41]. The AHI of 1.8 in our study might be a residual OSAS. Longitudinal follow-up is necessary to determine when recovery is maximal. More cases need to be examined to confirm the findings of the present study.

\section{Conclusion}

We reported a child initially suspected of having ADHD with drug resistant enuresis. Treatment of OSAS with adenotonsillectomy significantly improved snoring, enuresis, daytime behavioral problems, and neurocognitive functions. In order to identify pediatric OSAS, underlying neurodevelopmental disorders like ADHD, medical interview for snoring, and the oropharyngeal examination are important.

\section{Conflict of Interests}

The authors declare that there is no conflict of interests regarding the publication of this paper.

\section{References}

[1] T. Gislason and B. Benediktsdottir, "Snoring, apneic episodes, and nocturnal hypoxemia among children 6 months to 6 years old: an epidemiologic study of lower limit of prevalence," Chest, vol. 107, no. 4, pp. 963-966, 1995.

[2] N. J. Ali, D. Pitson, and J. R. Stradling, "Natural history of snoring and related behaviour problems between the ages of 4 and 7 years," Archives of Disease in Childhood, vol. 71, no. 1, pp. 74-76, 1994.

[3] C. L. Marcus, L. J. Brooks, K. A. Draper et al., "Diagnosis and management of childhood obstructive sleep apnea syndrome," Pediatrics, vol. 130, no. 3, pp. e714-e755, 2012.

[4] I. L. Westchester, International Classification of Sleep DisordersICSD, American Academy of Sleep Medicine, 2nd edition, 2005.

[5] S. J. Chang and K. Y. Chae, "Obstructive sleep apnea syndrome in children: epidemiology, pathophysiology, diagnosis and sequelae," Korean Journal of Pediatrics, vol. 53, no. 10, pp. 863-871, 2010.

[6] M. Greenfeld, R. Tauman, A. DeRowe, and Y. Sivan, "Obstructive sleep apnea syndrome due to adenotonsillar hypertrophy in infants," International Journal of Pediatric Otorhinolaryngology, vol. 67, no. 10, pp. 1055-1060, 2003.

[7] G. Polanczyk, M. S. de Lima, B. L. Horta, J. Biederman, and L. A. Rohde, "The worldwide prevalence of ADHD: a systematic review and metaregression analysis," American Journal of Psychiatry, vol. 164, no. 6, pp. 942-948, 2007.

[8] K. Sedky, D. S. Bennett, and K. S. Carvalho, "Attention deficit hyperactivity disorder and sleep disordered breathing in pediatric populations: a meta-analysis," Sleep Medicine Reviews, 2013.

[9] E. Ganelin-Cohen and A. Ashkenasi, "Disordered sleep in pediatric patients with attention deficit hyperactivity disorder: an overview," Israel Medicine Association Journal, vol. 15, pp. 705-709, 2013

[10] American Psychiatric Association, Diagnostic and Statistical Manual of Mental Disorders, Text Revision (DSM-IVIII-TR), Washington, DC, USA, American Psychiatric Association, 4th edition, 2000.

[11] A. Rechtschaffen and A. Kales, A Manual of Standardized Terminology, Techniques and Scoring System for Sleep Stages of Human Subjects, Brain Information Service, University of California, Los Angeles, Calif, USA, 1968.

[12] D. Wechsler, Manual for the Wechsler Intelligence Scale for Children-Revised, Psychological Corp., New York, NY, USA, 1974.

[13] A. S. Kaufman and N. I. Kaufman, Kaufman Assessment Battery for Children, American Guideline Service, Circle Pines, Minn, USA, 1983.

[14] P. A. Osterrieth, "The complex figure copy test," Archives of Psychology, vol. 30, pp. 206-256, 1994.

[15] A. Rey, L'examen clinique en psychologie, Presses Universitaires de France, Paris, France, 1964.

[16] T. M. Achenbach, Manual for Child Behavior Checklist 4-18 and 1991 Profile, Department of Psychiatry, University of Vermont, Burlington, Vt, USA, 1991.

[17] R. D. Chervin, J. E. Dillon, C. Bassetti, D. A. Ganoczy, and K. J. Pituch, "Symptoms of sleep disorders, inattention, and hyperactivity in children," Sleep, vol. 20, no. 12, pp. 1185-1192, 1997.

[18] R. D. Chervin and K. H. Archbold, "Hyperactivity and polysomnographic findings in children evaluated for sleepdisordered breathing," Sleep, vol. 24, no. 3, pp. 313-320, 2001.

[19] R. D. Chervin, K. H. Archbold, J. E. Dillon et al., "Inattention, hyperactivity, and symptoms of sleep-disordered breathing," Pediatrics, vol. 109, no. 3, pp. 449-456, 2002.

[20] L. M. O’Brien, C. R. Holbrook, C. B. Mervis et al., "Sleep and neurobehavioral characteristics of 5- to 7-year-old children with parentally reported symptoms of attention-deficit/hyperactivity disorder," Pediatrics, vol. 111, no. 3, pp. 554-563, 2003.

[21] L. M. O’Brien, A. Ivanenko, V. M. Crabtree et al., "Sleep disturbances in children with attention deficit hyperactivity disorder," Pediatric Research, vol. 54, no. 2, pp. 237-243, 2003.

[22] S. Cortese, S. V. Faraone, E. Konofal, and M. Lecendreux, "Sleep in children with attention-deficit/hyperactivity disorder: meta-analysis of subjective and objective studies," Journal of the American Academy of Child and Adolescent Psychiatry, vol. 48, no. 9, pp. 894-908, 2009.

[23] S. Naseem, B. Chaudhary, and N. Collop, "Attention deficit hyperactivity disorder in adults and obstructive sleep apnea," Chest, vol. 119, no. 1, pp. 294-296, 2001.

[24] R. D. Chervin, D. L. Ruzicka, K. H. Archbold, and J. E. Dillon, "Snoring predicts hyperactivity four years later," Sleep, vol. 28, no. 7, pp. 885-890, 2005.

[25] P. E. Brockmann, M. S. Urschitz, M. Schlaud, and C. F. Poets, "Primary snoring in school children: prevalence and 
neurocognitive impairments," Sleep and Breathing, vol. 16, no. 1, pp. 23-29, 2012.

[26] Y.-S. Huang, N.-H. Chen, H.-Y. Li, Y.-Y. Wu, C.-C. Chao, and C. Guilleminault, "Sleep disorders in Taiwanese children with attention deficit/hyperactivity disorder," Journal of Sleep Research, vol. 13, no. 3, pp. 269-277, 2004.

[27] L. J. Brooks and H. I. Topol, "Enuresis in children with sleep apnea," Journal of Pediatrics, vol. 142, no. 5, pp. 515-518, 2003.

[28] R. Brouilette, D. Hanson, R. David et al., "A diagnostic approach to suspected obstructive sleep apnea in children," The Journal of Pediatrics, vol. 105, no. 1, pp. 10-14, 1984.

[29] U. Çinar, C. Vural, B. Çakir, E. Topuz, M. I. Karaman, and S. Turgut, "Nocturnal enuresis and upper airway obstruction," International Journal of Pediatric Otorhinolaryngology, vol. 59, no. 2, pp. 115-118, 2001.

[30] D. Baeyens, H. Roeyers, L. D’Haese, F. Pieters, P. Hoebeke, and J. Vande Walle, "The prevalence of ADHD in children with enuresis: comparison between a tertiary and non-tertiary care sample," Acta Paediatrica, vol. 95, no. 3, pp. 347-352, 2006.

[31] D. Baeyens, H. Roeyers, P. Hoebeke, S. Verté, E. van Hoecke, and J. Vande Walle, "Attention deficit/hyperactivity disorder in children with nocturnal enuresis," Journal of Urology, vol. 171, no. 6, pp. 2576-2579, 2004.

[32] S. A. Kaufman and N. L. Kaufman, Kaufman Assessment Battery for Children: Interpretive Manual, Ministry of Education, Culture \& Sports, Jerusalem, Palestine, 1996.

[33] B.-C. Friedman, A. Hendeles-Amitai, E. Kozminsky et al., "Adenotonsillectomy improves neurocognitive function in children with obstructive sleep apnea syndrome," Sleep, vol. 26, no. 8, pp. 999-1005, 2003.

[34] L. J. Findley, J. T. Barth, D. C. Powers, S. C. Wilhoit, D. G. Boyd, and P. M. Suratt, "Cognitive impairment in patients with obstructive sleep apnea and associated hypoxemia," Chest, vol. 90, no. 5, pp. 686-690, 1986.

[35] B. Naegele, J.-L. Pepin, P. Levy, C. Bonnet, J. Pellat, and C. Feuerstein, "Cognitive executive dysfunction in patients with obstructive sleep apnea syndrome (OSAS) after CPAP treatment," Sleep, vol. 21, no. 4, pp. 392-397, 1998.

[36] M.-A. Bedard, J. Montplaisir, J. Malo, F. Richer, and I. Rouleau, "Persistent neuropsychological deficits and vigilance impairment in sleep apnea syndrome after treatment with continuous positive airways pressure (CPAP)," Journal of Clinical and Experimental Neuropsychology, vol. 15, no. 2, pp. 330-341, 1993.

[37] H. M. Engleman, R. N. Kingshott, S. E. Martin, and N. J. Douglas, "Cognitive function in the sleep apnea/hypopnea syndrome (SAHS)," Sleep, vol. 23, supplement 4, pp. S102-S108, 2000.

[38] K. Watanabe, T. Ogino, K. Nakano et al., "The Rey-Osterrieth Complex Figure as a measure of executive function in childhood," Brain and Development, vol. 27, no. 8, pp. 564-569, 2005.

[39] D. S. Lewin, R. C. Rosen, S. J. England, and R. E. Dahl, "Preliminary evidence of behavioral and cognitive sequelae of obstructive sleep apnea in children," Sleep Medicine, vol. 3, no. 1, pp. 5-13, 2002.

[40] C. L. Marcus, R. H. Moore, C. L. Rosen et al., "A randomized trial of adenotonsillectomy for childhood sleep apnea," The New England Journal of Medicine, vol. 368, no. 25, pp. 2366-2376, 2013.

[41] R. D. Chervin, D. L. Ruzicka, B. J. Giordani et al., "Sleepdisordered breathing, behavior, and cognition in children before and after adenotonsillectomy," Pediatrics, vol. 117, no. 4, pp. e769-e778, 2006. 


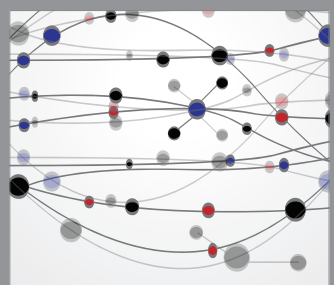

The Scientific World Journal
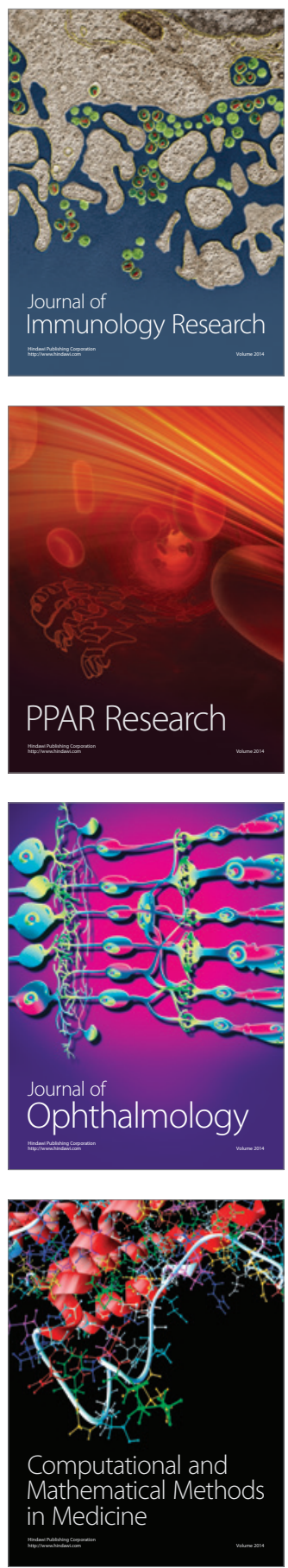

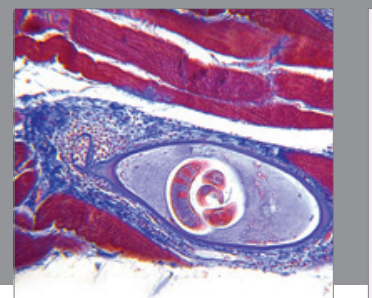

Gastroenterology

Research and Practice
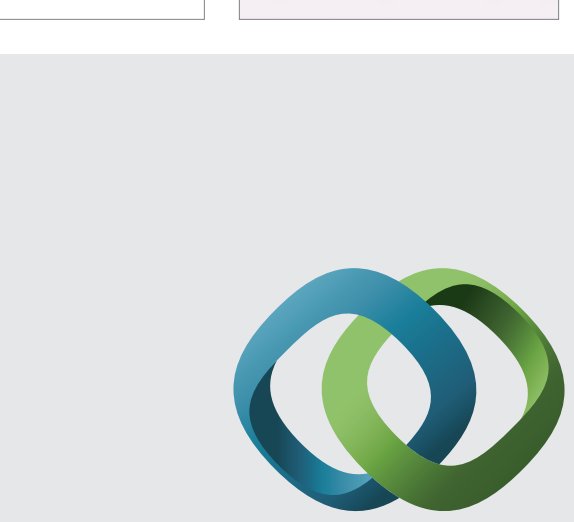

\section{Hindawi}

Submit your manuscripts at

http://www.hindawi.com
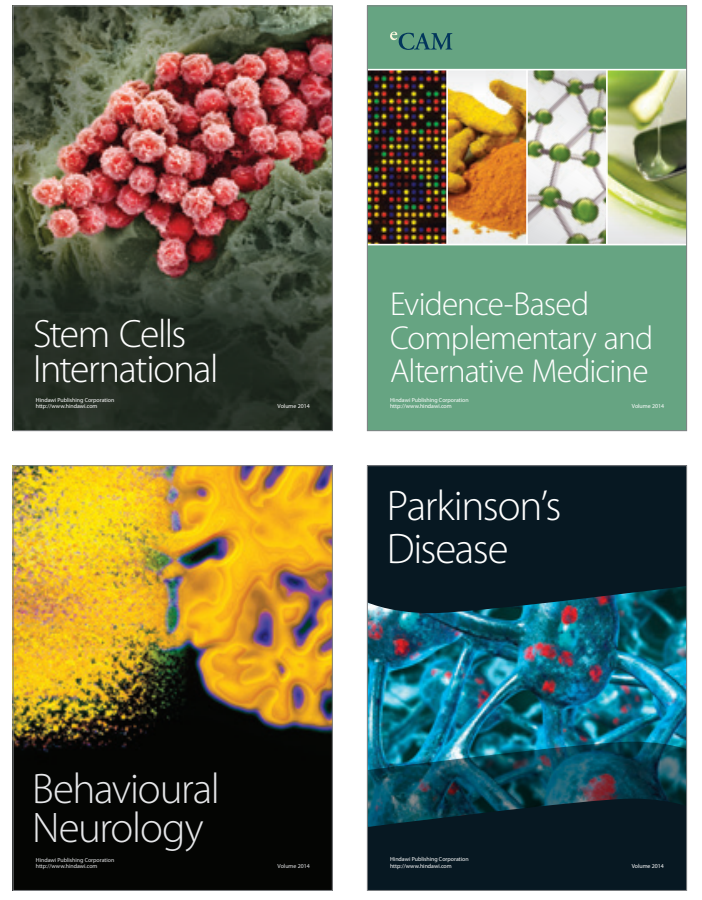
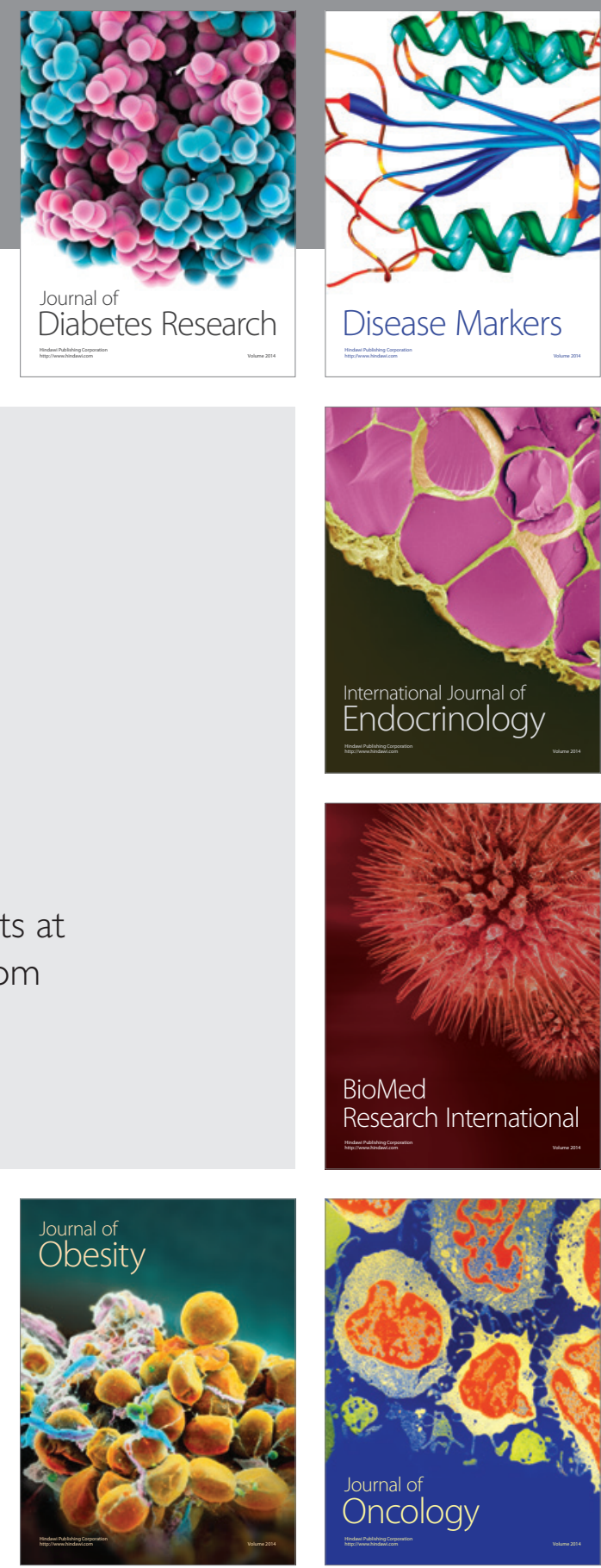

Disease Markers
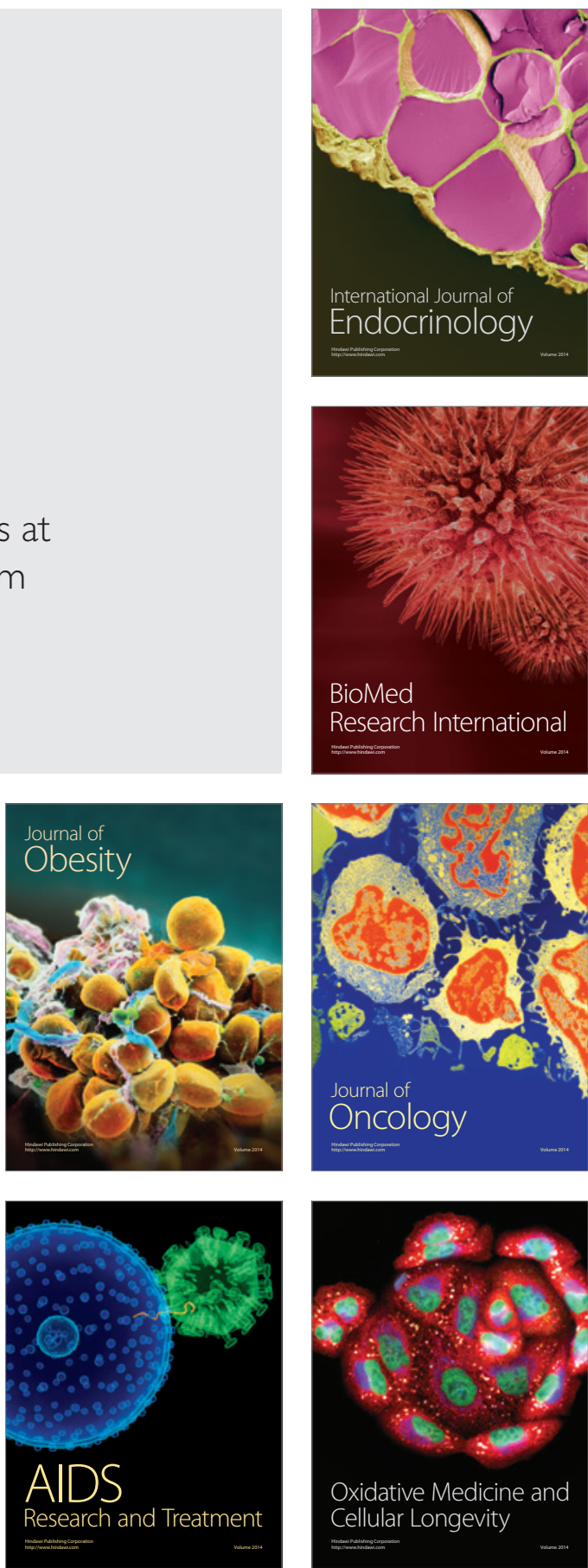